DOI: http://dx.doi.org/10.12957/demetra.2014.8502

\title{
Integração dos campos de saúde coletiva e alimentação e nutrição num contexto de promoção da saúde relacionado às doenças crônicas não transmissíveis
}

\author{
Integration of fields of public health and food and nutrition in the context of health promotion \\ related to chronic non-communicable diseases
}

\author{
Luana da Silva Baptista Arpini' \\ André Fiorin Arpini² \\ I Secretaria Estadual de Saúde do Espírito Santo. \\ Vitória, ES, Brasil. \\ ${ }^{2}$ Secretaria Municipal de Saúde de Cariacica. \\ Cariacica, ES, Brasil. \\ Correspondência / Correspondence \\ Luana da Silva Baptista Arpini \\ E-mail: luanaarpini@hotmail.com
}

\section{Resumo}

Considerando que as doenças crônicas não transmissíveis (DCNT) são um dos problemas mais relevantes na saúde pública na atualidade, este artigo pretendeu revisar criticamente a literatura quanto à integração entre os campos de Saúde Coletiva (SC) e Alimentação e Nutrição (AN) no contexto da promoção da saúde relacionado às DCNTs. Desta forma, evidenciou-se que no trato das questões referentes às DCNTs se faz necessário pensar diversos fatores que interagem formando um complexo, porém possível, processo de (re)construção de estratégias mais eficazes de intervenção, e que é preciso cada vez mais compor ações intersetoriais e interdisciplinares para resultados mais efetivos.

Palavras-chave: Alimentação. Nutrição em Saúde Pública. Promoção da Saúde. Doenças Crônicas.

\section{Abstract}

Whereas chronic non-communicable diseases (NCDs) are one of the most important problems in public health today, this article aims to critically review the literature regarding integration between the fields of Public Health (PH) and Food and Nutrition $(\mathrm{FN})$ in the context of health promotion related to NCDs. Thus, it became clear that in dealing with issues related to NCDs it is necessary to think several factors that interact to form a complex, but possible, process of the (re)constructing more effective 
intervention strategies, and that it becomes increasingly necessary to make up inter-sectoral and interdisciplinary actions aiming at more effective results.

Key words: Feeding. Nutrition. Public Health. Health Promotion. Chronic Disease.

\section{Introdução}

As doenças crônicas não transmissíveis (DCNTs) no Brasil foram responsáveis por $72 \%$ das mortes ocorridas em 2007, sendo consideradas um dos problemas mais relevantes, ${ }^{1}$ além de serem as que mais oneram custos para o sistema de saúde e que, não obstante, contribuem para a redução progressiva da qualidade de vida da população.

Dentre os múltiplos fatores preditores das DCNTs, estão: características biológicas, demográficas, socioeconômicas e sobretudo os hábitos de vida, como qualidade da alimentação e prática de atividade física. Em adição, fatores que marcam a contemporaneidade - tais como urbanização; mudanças na relação entre tempo e espaço; industrialização; multiplicidade de atribuições da mulher, que ainda exerce papel central no cuidado com a saúde e com a alimentação da família; o marketing, dentre outros - são atribuídos às mudanças nas práticas alimentares dos brasileiros. ${ }^{2}$

No trato das DCNTs e do sistema público de saúde do Brasil, a promoção de saúde tem ganhado notória importância. Dessa forma, é relevante ressaltar as políticas de alimentação e nutrição, bem como o acesso à alimentação adequada - em quantidade e qualidade -, o que torna indispensável traçar um elo desses determinantes com a condição de vida da população.

Assim, por muito tempo, a alimentação e a nutrição (AN), apesar de relevantes em todos os níveis de saúde e citadas em todas as políticas e legislações relacionadas à saúde e direitos humanos - inclusive na própria Constituição Federal, tida como Carta Magna para a população brasileira -, não detinham espaço delineado e soavam como uma profecia. Desta maneira, eram de conhecimento público e se discute a respeito, mas não se conseguia introduzi-las e inter-relacionálas no campo da Saúde Coletiva nos diversos níveis de atenção.

A Política Nacional de Alimentação e Nutrição (PNAN), ${ }^{3}$ publicada no final dos anos 90, marcou uma etapa importante para a configuração da área no campo da saúde, evidenciando as transições epidemiológica, nutricional e demográfica com a convivência, no país, de situações extremas de desnutrição e deficiências nutricionais, ao lado de altas prevalências de obesidade e doenças associadas à alimentação. ${ }^{4}$ 
Portanto, deve-se pensar a Alimentação / Nutrição também epidemiologicamente, sem reduzilas a relações de variáveis sociodemográficas e econômicas, procurando articular categorias (natureza e cultura) que possam ampliar os estudos, por exemplo, sobre riscos por problemas nutricionais, ou a interação entre a deficiência de micronutrientes e fatores socioeconômicos e culturais e outras relações. ${ }^{5}$

Dito isso, o elo possível entre AN como elementos de promoção de saúde e de cuidado no trato das DCNTs, embora incontestavelmente indispensável, é um tanto quanto frágil, devido a elementos envolvidos desde as dificuldades de implementação das políticas de alimentação e nutrição até os hábitos alimentares da população.

Considerando a relevância da temática para a saúde pública, o objetivo deste trabalho foi revisar criticamente a literatura, propondo discutir a interface entre os campos de Saúde Coletiva (SC) e Alimentação e Nutrição (AN) no contexto de promoção da saúde relacionado às DCNTs, delineando questões pertinentes ao acesso a alimentação, liberdade e possibilidade de escolha alimentar da sociedade; contextualizar os principais fatores que interagem nesse complexo, bem como evidenciar a contribuição das políticas específicas de AN para a concretização dos hábitos de vida saudáveis na prevenção de DCNTs.

\section{Métodos}

Realizou-se ampla busca de evidências científicas disponíveis no site oficial do Ministério da Saúde Brasil e na base de dados BIREME (incluindo LILACS, MEDLINE e SciELO), através da Biblioteca Virtual em Saúde (BVS) - BIREME/PAHO/WHO, sem utilização de filtros de data, idioma ou tipo de publicação, utilizando os descritores: "alimentação", "nutrição em saúde pública", "promoção da saúde" e "doença crônica"; congregando as informações pertinentes ao tema e promovendo o processo de atualização científica.

Como critério de elegibilidade, consideraram-se somente os artigos brasileiros, ou que reportassem a temática no Brasil, uma vez que cada país possui políticas, programas e legislações específicas e o objetivo deste estudo não contempla abrangências mundiais.

\section{Resultados e discussão}

A análise textual foi conduzida após seleção dos artigos que contemplassem a problemática deste estudo ( $n=14)$, cujos resultados estão apresentados a seguir, de forma descritiva por grupos temáticos emergentes. 


\section{A promoção da saúde}

O papel da promoção da saúde, em sua importância como estratégia fundamental para o enfrentamento dos problemas do processo saúde-doença-cuidado e da sua determinação, ${ }^{6}$ situase em processo de construção teórica e prática, e aponta para o redirecionamento das políticas e programas de saúde no país. ${ }^{7}$

Apesar de não ser recente, o termo "promoção da saúde" reaparece enquanto estratégia promissora para o campo da Saúde Pública somente nos últimos vinte anos, como resposta à crescente medicalização, à baixa eficácia dos serviços de saúde e aos altos custos do setor. ${ }^{7}$

Segundo Pádua \& Boog, ${ }^{8}$ quando se trata da promoção da saúde, torna-se evidente a importância do trabalho multiprofissional, que pressupõe a presença e a participação de vários profissionais da saúde, o que vai garantir, também, a integralidade na assistência. É preciso considerar, no entanto, que a integralidade na assistência não se configura como um processo automático, resultado direto da presença de diversos saberes e profissões. As ações de promoção da saúde impõem a criação de espaços democráticos e participativos, a fim de estabelecer uma aproximação com a realidade dos indivíduos e das populações. ${ }^{7}$ A "saúde seria o resultado de um amplo espectro associado com a qualidade de vida, compreendendo um conjunto de valores: justiça social, educação, renda, habitação, alimentação, nutrição, trabalho etc.””

É relevante ressaltar a forma como os profissionais de saúde e outros setores da sociedade interagem com este tema em especial. Em tese, é preciso considerar que as ações não podem e não devem se centrar nos serviços de saúde, ou estar centralizadas nos profissionais de saúde. Desta forma, intersetorialidade se apresenta como uma estratégia prioritária na construção de espaços legítimos para o diálogo entre os diferentes setores envolvidos na dinâmica alimentar, visando assegurar o acesso à alimentação digna, ${ }^{7}$ enquanto os profissionais de saúde se comprometem a trabalhar o saber integrado e compartilhado na prática de promoção da saúde.

Este não necessariamente é um processo automático condicionado apenas à presença de várias disciplinas diferentes. Com efeito, a interdisciplinaridade é um desafio que se impõe nos dias de hoje aos profissionais de saúde, que, reconhecendo os limites de cada área, buscam novas possibilidades de trabalho, ao mesmo tempo em que enfrentam barreiras ao trabalho conjunto, por razões de ordem histórica, social e profissional. ${ }^{8}$

Muitas vezes, o próprio modelo de atenção não favorece a concretização da promoção da saúde e a qualidade de vida, sendo focado em suprir demandas clínicas específicas, contradizendo a proposta do cuidado coletivo. Ambos devem caminhar juntos, mas é essencial que a ênfase seja dada a promoção e prevenção, e não ao assistencialismo. 


\section{Integração "Alimentação e Nutrição" e "Saúde Coletiva"}

A Saúde Coletiva (SC), como campo científico assumidamente interdisciplinar - um amplo leque temático que incursiona e fertiliza outros campos -, integra-se a questões alimentares e nutricionais, ${ }^{5}$ mas ainda que seja um campo demasiadamente fértil e abrangente, seu fazer inclusive no campo das pesquisas - não é um processo automático, mas dependente e relacionado com uma quantidade infindável de fatores.

Segundo Bosi \& Prado, ${ }^{5}$ três núcleos se fazem fortemente presentes e expressam a constituição epistemológica do campo, embora seja importante reconhecer um debate que se polariza entre a fragmentação e a interdisciplinaridade no campo: Epidemiologia, Ciência Humanas e Sociais e Política do Planejamento e Gestão de Sistemas de Saúde.

No âmbito da Epidemiologia, a Saúde Coletiva busca relações estatísticas entre "fatores causais" de doenças em populações e se concretiza num fortíssimo avanço de metodologias e técnicas no processo saúde/doença, como na Alimentação e Nutrição, com relação aos estudos sobre fome e obesidade no Brasil. ${ }^{5}$ Reflexo disso foi a elaboração da Política Nacional de Alimentação e Nutrição (PNAN), ${ }^{3}$ cujas evidências epidemiológicas nortearam a argumentação e definiram suas diretrizes. ${ }^{4}$

No que tange ao desdobramento da Saúde Coletiva enquanto ciência humana e social, fazem-se reflexões, críticas e transformações do conceito "pensar em saúde”. Sua contribuição maior talvez seja essa insistência na necessidade de ousar pensar a alimentação e nutrição como fenômenos sociais - contribuição construída no seio dessa imbricação: Alimentação e Nutrição em Saúde Coletiva. ${ }^{5}$

Apesar de inserida na dimensão de políticas, planejamento e gestão em saúde, a SC incorpora elementos conceituais enfatizando as relações com o Estado e suas crises, e a necessidade do planejamento e da gestão em âmbito populacional - essenciais inclusive em relação a Alimentação e Nutrição. ${ }^{5}$

Nesse sentido, implementada em 2006, a Política Nacional de Promoção da Saúde (PNPS $)^{9}$ incluiu uma série de ações em articulação intersetorial e desenvolvimento de políticas, educação em saúde, monitoramento de doenças e de fatores de risco e fornecimento de atenção à saúde centrada em dietas saudáveis, atividade física, redução do tabagismo e do uso prejudicial de álcool. ${ }^{1}$ Isso fortaleceu as ações da promoção das práticas alimentares saudáveis delineadas anteriormente na PNAN. ${ }^{3}$

Quando a Saúde Coletiva se relaciona, em especial com o campo da Alimentação, considerando que este, “por sua vez, constitui um 'processo' dinâmico de construção e reconstrução, seja de produção, consumo ou trabalho do homem na sociedade contemporânea" ${ }^{10}$ é relevante pensar o bojo de ações e considerações que tal relação fornece, tanto para o trato das DCNTs, como para a inserção da disciplina de Nutrição no leque que sustenta a Saúde Coletiva. 
Nesse contexto, a Saúde Coletiva é uma porta aberta para os estudos nutricionais (enfoque epidemiológico) e alimentares (enfoque das ciências humanas e sociais), ${ }^{11}$ e sugere um caminho promissor para o campo da Alimentação e Nutrição. ${ }^{7}$ Faz-se necessário, portanto, investir na aproximação, no confronto, nas contradições entre esses campos e núcleos de saberes e práticas, em busca da superação dialética dessa polarização, tal como exigem os desafios cada vez mais intensos que se apresentam para a saúde, alimentação e tantas outras esferas da vida humana. ${ }^{5}$

Pelos argumentos supracitados, evidencia-se a importância da integração da AN e SC, bem como suas respectivas políticas nos embates em saúde pública, inclusive na articulação com as organizações não governamentais, com o setor privado e com a sociedade civil.

\section{Contribuições da participação social}

É indispensável congregar a participação social em todas as questões que até aqui foram debatidas:

Uma vez que os indivíduos assumem responsabilidades sobre as consequências ambientais e sociais de suas escolhas cotidianas, a especificidade política da alimentação nas sociedades contemporâneas extrapola a esfera institucional (segurança alimentar e nutricional, desigualdades sociais no acesso à alimentação, políticas agrícolas e regulamentação da publicidade de alimentos) para atingir a esfera privada. ${ }^{12}$

Por este caminho, vale ressaltar que, dado o controle dos indivíduos sobre quaisquer fluxos e deslocamentos dos alimentos, pesam lógicas sociais, bem como convicções das mais diversas. Portanto, analisar as tangentes e desencadeamentos sociais das questões alimentares, desde a produção e comercialização dos produtos até seu consumo, é de extrema importância.

Ao admitirmos tal narrativa, é possível destacar que no momento em que os consumidores se dão conta dos riscos associados ao consumo de certos alimentos, ingressam na arena política que, no campo da Alimentação, se caracteriza pelo crescente número de escândalos alimentares e conflitos de controle e regulação, bem como pelo grande número de discursos conflituosos. ${ }^{12}$

Ao longo da história, e também em consonância com notabilidade e importância sócio-comercial, em relação a algumas necessidades alimentares especiais, parafraseando Burlandy, ${ }^{13}$ a influência das diversas organizações sociais nas políticas públicas é notável, bem como sua amplitude temática, e elas contribuíram para a construção de um lugar institucional e uma abordagem mais efetiva e visível nas questões alimentares e nutricionais. 
O fortalecimento das ações de promoção da saúde se deu junto à exigibilidade da garantia dos direitos humanos, inclusive ao que remete à alimentação adequada pela população. Assim, a atuação de movimentos e organizações sociais no campo da AN ganhou expressividade com a progressiva redemocratização do país, na década de $1980,{ }^{13}$ marcada pela criação do Sistema Único de Saúde brasileiro - o SUS.

A atuação da sociedade civil na área de AN foi fundamental para a consolidação tanto da PNAN, quanto da Política Nacional de Segurança Alimentar e Nutricional (PNSAN) ${ }^{13}$ Resultado de tal processo, recentemente, o Direito Humano à Alimentação Adequada (DHAA) se consolidou como emenda constitucional, ${ }^{14}$ devendo ser assegurado a todo brasileiro o acesso ao alimento seguro, em quantidade e qualidade, essenciais para sua sobrevivência.

Esta convergência em torno de uma agenda de promoção da alimentação adequada e saudável configura-se como elemento de diálogo propositivo entre movimentos da sociedade civil de Segurança Alimentar e Nutricional (SAN) e de Saúde Coletiva. ${ }^{4}$

Citada há pouco, como fruto de esforço conjunto, inclusive com a sociedade civil, a PNAN ${ }^{3}$ consagrou em termos legislativos os princípios do Direito Humano à Alimentação Adequada (DHAA) e da SAN como norteadores de suas ações. Em sua magnitude, direcionada à promoção da saúde e vigilância alimentar e nutricional, a PNAN, ${ }^{3}$ por sua vez, tem como base de informações o SISVAN (Sistema Nacional de Vigilância Alimentar e Nutricional). Oriunda da rotina da atenção básica de saúde, em especial das equipes de saúde da família, é atualmente utilizada para mensurar os resultados alcançados pelos gestores de saúde das esferas estadual e municipal no cumprimento das metas de alimentação e nutrição assumidas no Pacto Nacional de Saúde. ${ }^{4}$

\section{Incentivo às práticas alimentares saudáveis}

Ao considerarmos a promoção de saúde e o incentivo a práticas alimentares saudáveis na correlação com o que já foi explicitado até este momento, faz-se necessário adentrar um pouco as veredas das relações dinâmicas que os sujeitos estabelecem com a alimentação.

Parece-nos um tanto quanto lógico que, se é possível estabelecer relação entre a dinâmica social e alimentação, e estas refletirão na prevenção de morbi/mortalidades por DCNTs, é possível supor que esse processo deva acontecer no âmbito da educação nutricional e em saúde. Nesse contexto, a promoção de práticas alimentares saudáveis é uma estratégia de vital importância para o enfrentamento dos problemas alimentares e nutricionais do contexto atual, ${ }^{6} \mathrm{em}$ que se faz necessário estruturar um objeto atingível e passível de criação de estratégias de intervenção para promoção e prevenção em saúde, especialmente no que tange às DCNTs, em todos os níveis de atenção em que haja comprometimento mútuo dos profissionais, da sociedade e do poder público. 
Embora reconhecendo que estratégias de educação voltadas à saúde como um todo, especificamente a nutricional, não são suficientes, torna-se relevante um conjunto de estratégias que venham a fortalecer o impacto da questão alimentar nos diferentes setores sociais e governamentais, impelindo ações numa dimensão cada vez mais intersetorial e, portanto, mais abrangente.

Na esteira desta colocação, as propostas de inclusão dos temas de segurança alimentar nos projetos pedagógicos escolares, nos diferentes níveis de ensino, podem contribuir para a instrumentalização dos indivíduos, permitindo aos sujeitos "navegarem" nesse mar de informações e ainda, considerá-las dentro das discussões político-filosóficas do ensino brasileiro, não reduzindo o tema à mera inclusão de conteúdos. ${ }^{6}$

No entanto, isso não é suficiente para propor mudanças nos hábitos alimentares da população. O ser educador em nutrição não se resume a transmitir informações corretas de forma didática, porque implica apreender a maneira como o interlocutor vivencia o problema alimentar, não apenas em relação ao consumo alimentar propriamente dito, mas a todas as questões de natureza subjetiva e interpessoal imbricadas no comportamento alimentar. ${ }^{15}$

Educar para a alimentação saudável exige: confrontação de novas práticas com as representações sociais dos alimentos e com o significado simbólico deles para os sujeitos do processo educativo; a ressignificação dos alimentos; e a construção de novos sentidos para o ato de comer. Muitos profissionais de saúde se sentem incapacitados de adentrar esse processo educativo, uma vez que os cursos de graduação não embasam a temática, ou ainda, por descrédito na mudança de hábitos alimentares por não sabê-lo como fazê-lo e julgar ser utópico.

Tal situação é especialmente cronificada ao se considerar o caráter ainda muito clínico, individualista e pouco politizado, enfocado não apenas nos cursos de formação das diversas áreas de saúde, mas também nas frentes de formação do sujeito. Por exemplo, pode-se citar a mídia e a escola e a família, que ao tratarem maciçamente os assuntos de saúde como análogos exclusivos das doenças, contribuem significativamente para sujeitos e profissionais pouco abrangentes e apartados de questões e ações mais coletivas e de impacto amplo.

Outras ações são necessárias para a promoção da saúde e das práticas alimentares saudáveis. Providências políticas eficazes, como as fiscais, que priorizem o acesso da população a alimentos saudáveis, como frutas e vegetais, e desencorajem o consumo de alimentos processados; assim como intervenções que orientem o planejamento urbano com o objetivo de promover o estilo de vida ativo e a prática de atividade física. ${ }^{1}$

Ações para reduzir o teor de sódio dos alimentos são especialmente necessárias e vêm sendo trabalhadas através de legislações e políticas específicas, de implementação em longo prazo. Citase o Plano de Ações Estratégicas para o Enfrentamento das Doenças Crônicas Não Transmissíveis 
(DCNT), no Brasil 2011-2022, ${ }^{16}$ que como política pública, pretende direcionar ações em diversas frentes de atuação embora ainda seja prematuro avaliar suas possibilidades e impactos.

\section{Acesso ao alimento}

Apesar da importância da educação alimentar e nutricional na promoção de práticas alimentares saudáveis, ${ }^{6}$ se os problemas alimentares decorrem da dificuldade de acesso aos alimentos, pouco impacto terão as técnicas de aconselhamento e orientação alimentar. ${ }^{7}$

Dito isso, é preciso reconhecer a extensão territorial brasileira e sua história sócio-política marcada por profundas e extensas desigualdades sociais e econômicas. E ainda, reconhecer que é significativa a parcela populacional que encontra dificuldades na alimentação, mais especificamente em relação ao acesso ao alimento.

Segundo Recine \& Vasconcellos, ${ }^{4}$ monitorar a situação de saúde, alimentar e nutricional de maneira sistemática revela desigualdades entre regiões geográficas, grupos populacionais e populações vulneráveis, e permite subsidiar intervenções voltadas para a prevenção e o controle de distúrbios nutricionais e doenças associadas a alimentação e nutrição, e para a promoção de práticas alimentares e estilos de vida saudáveis.

Neste sentido, deve-se avançar em direção ao rompimento com o entendimento do SISVAN como simples coleta sistemática de dados antropométricos, uma vez que pode potencializar a articulação entre os setores de governo, fortalecer uma concepção ampliada da questão alimentar, nutricional e de saúde, além de contribuir para destinar os programas sociais aos grupos mais vulneráveis. ${ }^{17}$

Assim sendo, tomando como base os fundamentos norteadores das diretrizes da PNAN, ${ }^{3}$ podese concluir que a efetivação da vigilância, capaz de ultrapassar a abordagem do estado nutricional abrangendo a dimensão alimentar, demanda também assegurar a disponibilidade e as condições de acesso social e físico aos alimentos, servindo como base para o planejamento e tomada de decisão. ${ }^{17}$

Os programas governamentais de transferência direta de renda, como o Programa Bolsa Família (PBF), ${ }^{18}$ vêm possibilitando a aquisição dos alimentos pela sociedade e contribuindo para a redução da desnutrição no Brasil. Isso, porém, não garante que a população consuma alimentos que sejam adequados a suas necessidades.

Se por um lado há o $\mathrm{PBF},{ }^{18}$ por outro há a emancipação e a captura dos padrões culturais alimentares dessa família. Assim, o relativo crescimento da liberdade de escolha dos consumidores é conectado a uma busca pela emancipação, identidade pessoal e autonomia na esfera privada, 
apontando para novas formas de ação política. ${ }^{12}$ Nesse contexto, as ações intersetoriais que englobam não só os serviços de saúde, mas também as ações de incentivo à agricultura familiar, a hortas comunitárias e cooperativas agrícolas, se apresentam como alternativas viáveis. ${ }^{7}$

Desta forma, se o campo da alimentação é politizado através das políticas de produção agrícola e das políticas distributivas e nutricionais, cabe destacar que este processo de politização também se dá a partir da percepção dos riscos e da possibilidade de uso político das escolhas de consumo. ${ }^{12}$

\section{Publicidade de alimentos}

As questões referentes a educação alimentar, padrões de consumo alimentar e políticas públicas não estão esgotadas no universo da alimentação, nem suas interações. É preciso deliberar e problematizar sobre mais um importante tópico referente à alimentação - a saber, a publicidade de alimentos.

Se por um lado, a dinâmica cotidiana de vida favorece a demanda por produtos práticos e de rápido preparo e consumo, por outro há também uma preocupação social com a saúde diante dos múltiplos riscos das práticas alimentares contemporâneas associadas às doenças crônicas não transmissíveis, seja pelo baixo consumo de frutas, legumes e verduras e consequentemente de micronutrientes, ou pelo consumo excessivo de açúcar, sódio e gorduras. ${ }^{2}$

Como integrante do universo alimentar e com relação direta ao sistema econômico, a publicidade de alimentos sofre então inúmeras pressões e se metamorfoseia de acordo com as variações de mercado, que, por sua vez, interagem diretamente na captura e formação de padrões alimentares.

Considerando que o alimento é fruto de hábitos e costumes influenciados e reforçados por informações que subsidiam a tomada de decisão, a influência da mídia sobre a consolidação de uma orientação alimentar e nutricional da população mostra-se como temática relevante para investigação. ${ }^{19}$ Finalmente, a dimensão da comida como natureza envolve uma imagem idealizada desta última, oposta à cultura e tecnologia, que captura nossa imaginação do que é bom, saudável, verdadeiro e autêntico. ${ }^{12}$ Assim sendo, a forma como os valores sobre a alimentação são disseminados na sociedade, considerando a importância crescente que a cultura midiática vem assumindo neste processo, deve ser tratada como uma questão de caráter público. ${ }^{2}$

Devemos portanto levar em consideração que, embora não seja fator único ou determinante por si só, o aumento do tempo de exposição das pessoas ao conteúdo divulgado pela TV pode influenciar suas escolhas e seus hábitos alimentares, contribuindo para adesão a costumes alimentares saudáveis que promovam a saúde, ou para intensificação do sedentarismo e do consumo inadequado de alimentos. ${ }^{19}$ 
Pensando nesta questão, devemos ainda observar que, num sistema econômico que visa lucro, formação de capital, as estratégias comerciais em torno da alimentação e a forma como são compostas e a velocidade com que mudam, são também fatores que influenciam o padrão alimentar e, por que não, o acesso ao alimento, quando pensamos em padrão alimentar associado ao estilo de vida nesta dinâmica econômica e nas prioridades que pregam o mesmo sistema.

As estratégias de compras se transformaram, passando das compras cotidianas para as compras planejadas, em longo prazo. Além disso, as estratégias de marketing, como promoções, brindes e sorteios, e de publicidade das marcas, buscaram incentivar o consumo, intensificando com isso, as compras por impulso. ${ }^{20}$

Segundo Victora et al., ${ }^{21}$ desde a década de 1980, séries e outros programas de televisão têm sido utilizados intensivamente pelo governo, por organizações internacionais e da sociedade civil, a fim de promover comportamentos saudáveis, e, embora tais estratégias tenham surtido notório efeito, por outro lado, os meios de comunicação de massa têm sido amplamente utilizados também para anunciar, dentre outros, bebidas alcoólicas e comidas não saudáveis. A situação é ainda mais grave em relação às comidas processadas com alto teor calórico e poucos nutrientes, pois não estão sujeitas a qualquer tipo de regulação, nem mesmo em relação a propagandas para crianças e adolescentes.

A publicidade e propaganda utilizam estratégias inesgotáveis para persuadir a população ao consumo dos produtos anunciados, principalmente ao se tratar do público materno/infantil.

Os produtos alimentícios, em sua maioria com alta densidade energética, são divulgados na televisão, sobretudo de forma repetitiva e intensa, conjugados a imagens de personagens e/ou brindes, inclusive colecionáveis. Ressalta-se o valor nutritivo, explora a praticidade e transmite confiança de que aquele produto supre as necessidades alimentares; o intervalo comercial, muitas vezes, apresenta volume sonoro mais alto que o restante da programação, associado à expressão de afeto, alegria e musicalidade; algumas vezes sugerindo consumo excessivo ou substituição de alimentos naturais e saudáveis. ${ }^{2}$

Em estudo realizado por Santos et al. ${ }^{19}$ com o objetivo de analisar a quantidade e o horário das propagandas veiculadas pela TV sobre produtos alimentícios, classificar esses de acordo com a pirâmide alimentar e identificar o conteúdo calórico dos alimentos mais anunciados, por meio das informações nutricionais presentes nos rótulos, foi observado que das 239 propagandas alimentícias divulgadas pelas duas emissoras observadas; $85 \%$ anunciavam produtos contendo açúcares, óleos e gorduras, elevados teores de sódio nos alimentos, com destaque ao embutido e a batata frita; não foi identificado nenhum comercial abordando o consumo de frutas e hortaliças, sendo possível detectar que a mídia televisiva não tem contribuído para um estilo de vida saudável. 
Henriques et al. ${ }^{2}$ observaram ainda que se o regulamento proposto na consulta pública no 71/2006 estivesse em vigor, 91,6\% das propagandas de produtos alimentícios estariam em desacordo quanto à mensagem de alerta sobre os perigos do consumo excessivo de açúcar, gordura saturada, gordura trans e sódio. Esta mensagem deveria aparecer após a propaganda dos produtos ricos em tais nutrientes nocivos à saúde e às regras de apresentação da mensagem de alerta, permitindo perfeita legibilidade, visibilidade, além de ser perfeitamente audível, impedindo que o consumidor conhecesse e avaliasse o risco ao decidir sobre o consumo. E ainda, metade das propagandas estimulava o consumo excessivo dos alimentos anunciados.

A constatação inevitável do desafio de atuar no cenário descrito é assustadora, não só pela quantidade de frentes nas quais é necessário atuar, mas também pela disparidade aparente de velocidade com as quais as ações se apresentam. Isso ocorre, um pouco, pelo fato de que o modelo de ação historicamente é quase sempre baseado na resposta "curativa”, e não na promoção de ações duradouras e saudáveis.

Por outro lado, com o avanço da tecnologia e a rápida disseminação das informações em todos os aspectos, inclusive quanto a qualidade de vida, promoção da saúde e prevenção de doenças através da alimentação, a população busca respostas aos seus anseios. Ou seja, preocupa-se não só com o prazer que aquele alimento lhe traz, mas também desperta a necessidade de saber se o que está sendo consumido suprirá suas necessidades sem acarretar danos à saúde. Na última década, a preocupação com a alimentação vem conquistando grande espaço nas famílias brasileiras. Viria daí a obsessão pelo selo de qualidade, pela garantia da origem e da pureza original dos alimentos, criando ou recriando, mais ou menos magicamente, um laço entre o produto e sua origem, rompendo a barreira simbólica da embalagem. ${ }^{20}$

É possível perceber, entretanto, que a sociedade civil brasileira não se pronunciou quanto à promoção comercial agressiva de bebidas alcoólicas e comidas processadas não saudáveis, possivelmente pelo fato de que os danos para a saúde causados pelo consumo moderado de bebidas alcoólicas e alimentos industriais não estejam tão bem documentados e disseminados, não só nacional, mas globalmente, quanto aqueles referentes ao tabaco, por exemplo. ${ }^{21}$

Vale salientar ainda que a população desconhece e os estabelecimentos de comercialização não divulgam a obrigatoriedade da venda não casada dos brindes que são comercializados ou distribuídos a um preço mais baixo, conjugados à compra de produtos alimentícios. ${ }^{2}$ Assim, quando comparados à influência de escolas e familiares com a da mídia televisiva, é possível identificar que os comerciais possuem influência mais duradoura na medida em que utilizam mensagens persuasivas, atraentes e marcantes. ${ }^{2}$ Mas esse fenômeno não é um "privilégio" da atualidade, pois as subjetividades "pré-capitalísticas" já engendravam os chamados equipamentos coletivos de subjetivação. ${ }^{22}$ 
Isso resumidamente significa que, ao produzirmos discursos e práticas maciças e atraentes que valorizam e exaltam como boas formas de vida uma alimentação deficiente e/ou de composição não saudável, além da baixa quantidade/falta de estímulos contrários tão ou mais convincentes que os primeiros, isso requer que nos perguntemos: que subjetividades estamos produzindo? Que tipo de desejos e padrões de consumo estamos produzindo?

Embora não seja uma relação direta e obrigatória, e que não sejam as mídias e o capitalismo os únicos culpados, por assim dizer, a epidemiologia das DCNTs já descritas neste artigo demonstra que as perguntas sugeridas no parágrafo acima estão sendo feitas com considerável atraso.

Estas constatações não só são preocupantes, mas sugerem também que devemos então modificar nossas estratégias de promoção de hábitos alimentares saudáveis para torná-las igualmente atraentes e persuasivas. Para tal, é preciso também angariar esforços para diversificar as formas de intervenção e acompanhar a velocidade das campanhas publicitárias, ao mesmo tempo em que estas também devem se tornar alvo de intervenção para reduzir seu poder - ou seja, sua regulação.

\section{Considerações finais}

Como discorrido até aqui, são diversos os fatores que influenciam as questões relacionadas ao trato das doenças crônicas não transmissíveis e a efetivação de políticas de promoção e prevenção. Destaca-se que, embora seja impossível abarcar todos os preditores, é possível compreender e traçar estratégias eficazes em saúde e educação. Assim sendo, é relevante que objetivo de promover a saúde, especialmente no Brasil, não será alcançado sem que ações efetivas na área da vigilância e da assistência alimentar e nutricional sejam implementadas de forma articulada a um sistema de vigilância à saúde. ${ }^{23}$

Ao entendermos que a promoção e prevenção de saúde, em especial de educação nutricional e para saúde, não se dão sem interação setorial e interdisciplinar, podemos afirmar que se fazem necessárias ações que envolvam desde a revisão dos currículos de formação em saúde, passando por incentivos em especial na atenção primária à saúde; revisão dos currículos e práticas escolares desde a educação infantil até o ensino médio; incentivos fiscais e de produção de produtos mais saudáveis e com maior valor nutricional; e ações de regulação de exposição e venda de produtos como embutidos e congelados, por exemplo, que facilitem o acesso à informação nutricional e seus riscos à saúde do consumidor de forma mais efetiva.

Finalmente, desemboca-se em ações de regulação do mercado alimentar, não só no controle de SAN mas no que tange ao fator publicitário, este último em caráter emergencial. 
[...] Desta forma, mecanismos de proteção devem ser garantidos pelo Estado para que coíbam as estratégias que atuam sobre os aspectos subjetivos das práticas sociais e de valores sociais que inclusive afetam as relações sociais cabe à sociedade em geral, aos educadores e profissionais de saúde reiterarem a demanda pela propaganda ética e pela promoção de uma alimentação saudável através da mídia televisiva. ${ }^{2}$

Ao finalizar este escrito, é importante salientar, mais uma vez, que o maior desafio aparente nessas situações é o da interação ética e responsável entre os setores envolvidos. Este, embora óbvio, é essencialmente mais desgastante e problemático porque não deixa de estar relacionado com todos os fatores que influenciam na temática principal deste artigo.

\section{Referências}

1. Schmidt MI, Duncan BB, Silva GA, Menezes AM, Monteiro CA, Barreto SM, et al. Chronic noncommunicable diseases in Brazil: burden and current challenges. The Lancet 2011; 377(9781):1949-61.

2. Henriques P, Sally EO, Burlandy L, Beiler RM. Regulation of publicity for children's food as a strategy for promotion of health. Cien. Saúde Colet. 2012; 17(2):481-90.

3. Brasil. Ministério da Saúde. A Política Nacional de Alimentação e Nutrição (PNAN) [Internet]. Brasília: Ministério da Saúde; 2012 [acesso em 17 mar 2013] Disponível em: http://189.28.128.100/ nutricao/docs/geral/pnan2011.pdf

4. Recine E, Vasconcellos AB. National policies and the field of food and nutrition in collective health: the current scenario. Cien. Saúde Colet. 2011; 16(1):73-9.

5. Bosi MLM, Prado SD. Food and nutrition in public health: constitution, contours and scientific status. Cien. Saúde Colet. 2011; 16(1):7-17.

6. Santos LAS. Food and nutrition education in the context of promoting healthy food practices. Rev. Nutr. 2005; 18(5):681-92.

7. Ferreira VA, Magalhães R. Nutrition and health promotion: recent perspectives. Cad. Saúde Pública 2007; 23(7):1674-81.

8. Pádua JG, Boog MCF. Evaluation of inserting a nutritionist in the Primary Health Network of the municipalities located in the Metropolitan Region of Campinas, São Paulo, Brazil. Rev. Nutr. 2006 Ago;19(4):413-24.

9. Brasil. Ministério da Saúde. Política Nacional de Promoção da Saúde (PNPS) [Internet]. Brasília: Ministério da Saúde; 2010. [acesso em 17 mar. 2013.] Disponível em:http://bvsms.saude.gov.br/bvs/ publicacoes/politica_nacional_promocao_saude_3ed.pdf

10. Carvalho MCVS, Luz MT, Prado SD. Eating, nourishment and nutrition: instrumental analytic categories in the scientific research field. Cien. Saúde Colet. 2011; 16(1):155-63. 
11. Nunes ED. (Inter)disciplinary spaces: Food/Nutrition/Health/Public Health. Cien. Saúde Colet. 2011; 16(1):18-20.

12. Portilho F, Castañeda M, Castro IRR. Food in the contemporary context: consumption, political action and sustainability. Cien. Saúde Colet. 2011; 16(1):99-106.

13. Burlandy L. The role of civil society in building the field of Food and Nutrition in Brazil: elements for reflection. Cien. Saúde Colet. 2011; 16(1):63-72.

14. Brasil. Emenda Constitucional n $n^{\circ}$ 64. Altera o art. $6^{\circ}$ da Constituição Federal, para introduzir a alimentação como direito social [Internet]. 2010 Fev. 4. [acesso em 17 mar. 2013]. Disponível em: http://www.planalto.gov.br/ccivil_03/constituicao/Emendas/Emc/emc64.htm

15. Boog MCF. Atuação do nutricionista em saúde pública na promoção da alimentação saudável. Rev. Ciênc. Saúde 2008; 1:33-42.

16. Brasil. Ministério da Saúde. Plano de Ações Estratégicas para o Enfrentamento das Doenças Crônicas Não Transmissíveis (DCNT) no Brasil 2011-2022. Brasília: Ministério da Saúde; 2011. 145 p.

17. Machado NMV, Viteritte PL, Goulart DAS, Pinheiro ARO. Reflexões sobre saúde, nutrição e a estratégia de saúde da família [Internet]. 2006. [acessado em 17 mar. 2013]. Disponível em: http:// nutricao.saude.gov.br/docs/geral/noticia_01_09_06.pdf

18. Brasil. Lei no 10.836, de 9 de janeiro 2004. Cria o Programa Bolsa Família e dá outras providências. Diário Oficial da União 12 jan. 2004.

19. Santos CC, Stuchi RAG, Arreguy-Sena C, Pinto NAVD. A influência da televisão nos hábitos, costumes e comportamento alimentar. Cogitare Enferm. 2012; 17(1):65-71.

20. Fonseca AB, Souza TSN, Frozi DS, Pereira RA. Dietary modernity and food consumption: socioanthropological contributions to research in nutrition. Saúde Colet. 2011; 16(9):3853-62.

21. Victora CG, Barreto ML, Carmo Leal M, Monteiro CA, Schmidt MI, Paim J, et al. Health conditions and health-policy innovations in Brazil: the way forward. The Lancet 2011; 377(9782):2042-53.

22. Peres RS, Borsonello EC, Peres WS. The Schizoanalysis and the subjectivity production: practical and theoretical considerations. Psicologia em Estudo 2000; 5(1):35-43.

23. Assis AMO, Santos SMC, Freitas MCS, Santos JM, Silva MCM. The Brazilian Family Health Program: contributions to a discussion about the inclusion of the nutritionist in the multidisciplinary team. Rev. Nutr. 2002; 15(3):255-66.(3):255-66.

Recebido: 08/12/2013

Revisado: $24 / 4 / 2014$

Aprovado: $16 / 9 / 2014$ 
